Supporting Information

\title{
Rational Design of Electrolytes for Long-Term Cycling of Si Anodes over a Wide Temperature Range
}

Sujong Chae, ${ }^{\dagger}$ Won-Jin Kwak, ${ }^{, \S}$ Kee Sung Han, ${ }^{\ddagger}$ Shuang Li, ${ }^{\ddagger}$ Mark H. Engelhard, ${ }^{\ddagger}$ Jiangtao Hu, ${ }^{\dagger}$ Chongmin Wang, ${ }^{\ddagger}$ Xiaolin $\mathrm{Li}{ }^{*}$ and Ji-Guang Zhang ${ }^{{ }^{*}}$

† Energy and Environment Directorate, Pacific Northwest National Laboratory, Richland, Washington 99354, United States

‡ Environmental Molecular Sciences Laboratory, Pacific Northwest National Laboratory, Richland, Washington 99354, United States

$\S$ Current address: Department of Chemistry, Ajou University, Suwon, 16499, Republic of Korea

AUTHOR INFORMATION

Corresponding Author

* Corresponding authors. Email: jiguang.zhang@pnnl.gov (J.-G. Zhang), xiaolin.li@pnnl.gov (X.L. Li) 


\section{Experimental Description}

\section{Electrolyte preparation}

Battery-grade $\mathrm{LiPF}_{6}$ and lithium bis(fluorosulfonyl)imide (LiFSI) were purchased from BASF and Nippon Shokubai, respectively, and dried overnight at $120^{\circ} \mathrm{C}$ before use. Ethylene carbonate, ethyl methyl carbonate, vinylene carbonate (VC), and fluoroethylene carbonate $(\mathrm{FEC})$ were purchased from Gotion. OTE $(1 \mathrm{H}, 1 \mathrm{H}, 5 \mathrm{H}$-Octafluoropentyl 1,1,2,2tetrafluoroethyl ether) (99\%) was ordered from SynQuest Laboratories. All solvents were dried with molecular sieves (Sigma-Aldrich). OL electrolytes were prepared in an Ar-filled glove box (water and oxygen contents $<0.1 \mathrm{ppm}$ ) according to the molar ratio presented in Table S1.

\section{Electrochemical characterization}

The Si/graphite anode was prepared by casting a slurry of $88 \%$ Si/graphite composite (BTR New Energy Materials Inc.), 10\% polyimide binder, and 2\% carbon black (C65, Imerys) onto a copper foil. The cathode consisted of 90 wt $\%$ NMC532 (Toda), 5 wt $\%$ C45 carbon (Timcal) and 5 wt\% 5130 polyvinylidene difluoride (PVDF, Solvay) binder was provided by the CAMP (Cell Analysis, Modeling, and Prototyping) facility of Argonne National Laboratory. The mass loading for the Si/graphite anode and NMC532 cathode were $2.6 \mathrm{mg} / \mathrm{cm}^{2}$ and $11.4 \mathrm{mg} / \mathrm{cm}^{2}$, respectively. The anode and cathode were punched into disks with diameters of $15 \mathrm{~mm}$ and $14.28 \mathrm{~mm}$ (9/16 inch), respectively. The anode disks were heat treated at $300^{\circ} \mathrm{C}$ under argon flow for 2 hours to cure the polyimide. The cathode disks were vacuum dried at $120^{\circ} \mathrm{C}$ overnight.

Electrochemical tests were performed using CR2032-type coin cells on Land BT2000 battery testers at $30^{\circ} \mathrm{C}$ in environmental chambers. The electrolyte volume was 
controlled at $60 \mu \mathrm{L}$ for all coin-cell full cells. Al-clad stainless-steel cans (purchased from MTI, Inc.) with an additional Al foil disk (19 $\mathrm{mm}$ in diameter) were used to avoid corrosion of the stainless steel by LiFSI-based electrolytes when the cells were charged above 4.0 V. Polyethylene separators were used for conventional carbonate electrolyte and localized high concentration electrolytes (LHCEs). The half-cell tests were performed in the voltage range from 0.01 to $3.0 \mathrm{~V}$ for the first cycle and from 0.01 to $1.0 \mathrm{~V}$ for the further cycling test. The half-cells were cycled at $0.05 \mathrm{C}(1 \mathrm{C}=1000 \mathrm{~mA} / \mathrm{g})$ in constant-voltage mode for lithiation $(0.01 \mathrm{C}$ cutoff) for the first cycle, and at $0.33 \mathrm{C}$ (for both charging and discharging) in constant-voltage mode at the lithiation cutoff $(0.01 \mathrm{C})$ for the cycling tests. Before the full-cell tests, the Si/graphite anodes were prelithiated by cycling in a half-cell at $0.1 \mathrm{C}$ between 0.02 and $1.5 \mathrm{~V}$ three times, and then harvested. The full-cell tests were performed in the voltage range of 3.0 to $4.1 \mathrm{~V}$. The full cells were cycled at $0.05 \mathrm{C}$ $(1 \mathrm{C}=120 \mathrm{~mA} / \mathrm{g})$ for the first three cycles, then at $0.33 \mathrm{C}$ (for both charging and discharging) for the further cycling tests.

\section{Characterization}

The ionic conductivity was evaluated using a BioLogic MCS 10 analyzer with platinized conductivity cells. The viscosity was measured by a Brookfield DVII + Pro viscometer, which was equipped with a circulating bath for temperature control. For all the postmortem analyses, including scanning electron microscopy (SEM), electrode swelling, transmission electron microscopy (TEM), and x-ray photoelectron spectroscopy (XPS), all the electrodes were disassembled in the delithiated state and rinsed with dimethyl carbonate (DMC) in an Ar-filled glove box. Structural investigation of cycled Si/graphite anodes was carried out with SEM (Helios Nanolab dual-beam focused ion beam SEM, 
FEI). The sample preparation for cross-sectioned electrodes was performed by an ion mill (cooling cross-section polisher, JEOL). Electrode swelling in the delithiated state was estimated with the thickness change measured by a micrometer (Mitutoyo). TEM analysis was carried out with an FEI aberration-corrected Titan 80-300 S/TEM. XPS analysis of the electrodes was performed on a Physical Electronics Quantera scanning x-ray microprobe with a focused monochromatic Al Ka x-ray source.

\section{Pulsed-Field Gradient Nuclear Magnetic Resonance (PFG-NMR) Measurements}

To obtain the solvation structures and dynamic properties of a series of LiFSI/DMC electrolytes with the additives VC, FEC, and OTE, the diffusion coefficients $(D)$ of all components were determined. We used ${ }^{7} \mathrm{Li},{ }^{19} \mathrm{~F}$, and ${ }^{1} \mathrm{H}$ PFG-NMR for $\mathrm{Li}^{+}$cations, $\mathrm{FSI}^{-}$ anions, and protonated species (DMC and VC), respectively, at Larmor frequencies of $2 \pi \times 232.954,2 \pi \times 564.540$, and $2 \pi \times 599.415 \mathrm{rad} \cdot \mathrm{MHz}$, respectively, at $25^{\circ} \mathrm{C}$. For FEC and OTE diffusion measurements, the ${ }^{19} \mathrm{~F}$ PFG-NMR measurements were performed at a Larmor frequency of $2 \pi \times 563.938 \mathrm{rad} \cdot \mathrm{MHz}$. All measurements were carried out on a $600 \mathrm{MHz} \mathrm{NMR}$ spectrometer (Agilent, USA) equipped with a $5 \mathrm{~mm}$ z-gradient probe (Doty Scientific, USA), that can generate a maximum gradient strength of $\sim 31 \mathrm{~T} / \mathrm{m}$. The bipolar gradient PFG sequence (Dbppste, vendor supplied sequence in VNMRJ, Agilent, USA) was used to obtain PFG echo profiles with 16 equal steps as a function of gradient strength $(g)$; maximum gradient strengths were carefully chosen for observing the full decay of the echo profiles. Diffusion coefficients were determined from these echo profiles fitted with the Stejskal-Tanner equation,

$$
S(g)=S(0) \exp \left[-D(\gamma \delta g)^{2}\left(\Delta-\frac{\delta}{3}\right)\right]
$$


where $S(g)$ and $S(0)$ are the echo heights at the gradient strengths of $g$ and 0 , respectively, $D$ is the diffusion coefficient, $\mathrm{y}$ is the gyromagnetic ratio of the observing nucleus $\left({ }^{7} \mathrm{Li},{ }^{1} \mathrm{H}\right.$, or $\left.{ }^{19} \mathrm{~F}\right), \delta$ is the gradient length, and $\Delta$ is the diffusion delay. The $90^{\circ}$ pulse lengths for ${ }^{7} \mathrm{Li}$ and ${ }^{19} \mathrm{~F} /{ }^{1} \mathrm{H}$ were 8 and $11 \mu \mathrm{s}$, respectively. The detailed PFG-NMR parameters are summarized in Table S2. 
Table S1. Molar ratios of various OL electrolytes.

\begin{tabular}{|l|c|c|c|c|c|c|c|}
\hline \multicolumn{1}{|c|}{ OL } & 1 & 2 & 3 & 4 & 5 & 6 & 7 \\
\hline LiFSI & 0.51 & 0.51 & 0.51 & 0.51 & 0.51 & 0.51 & 0.51 \\
\hline DMC & 1.1 & 1.1 & 1.1 & 1.1 & 0.84 & 0.84 & 0.84 \\
\hline OTE & 3.3 & 2.2 & 1.65 & 1.1 & 0.84 & 0.42 & 0.21 \\
\hline FEC & \multicolumn{7}{|l}{} \\
\hline VC & \multicolumn{7}{l}{} \\
\hline
\end{tabular}

Table S2. PFG-NMR parameters used in this study.

\begin{tabular}{|l|c|c|c|c|c|c|}
\hline & $\Delta(\mathrm{ms})$ & $\delta(\mathrm{ms})$ & $\gamma / 2 \pi(\mathrm{MHz})$ & $\begin{array}{c}g_{\max } \\
(\mathrm{T} / \mathrm{m})\end{array}$ & Delay $(\mathrm{s})$ & $\mathrm{PW} 90^{\circ}(\mu \mathrm{s})$ \\
\hline${ }^{7} \mathrm{Li}$ & 30 & 2 & 16.546 & $7.0-10.0$ & 2 & 8 \\
\hline${ }^{1} \mathrm{H}$ & 30 & 2 & 42.577 & $1.2-2.7$ & 2 & 11 \\
\hline${ }^{19} \mathrm{~F}$ & 20 & 2 & 40.052 & $2.3-2.9$ & 2 & 11 \\
\hline
\end{tabular}




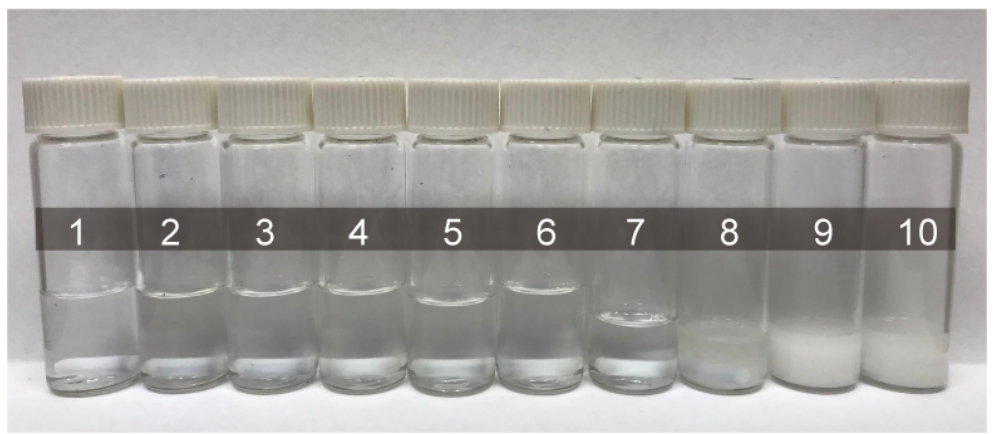

Figure S1. Digital photograph of various OL electrolytes. OLs 8, 9, and 10 were rendered semi opaque by precipitated salt.

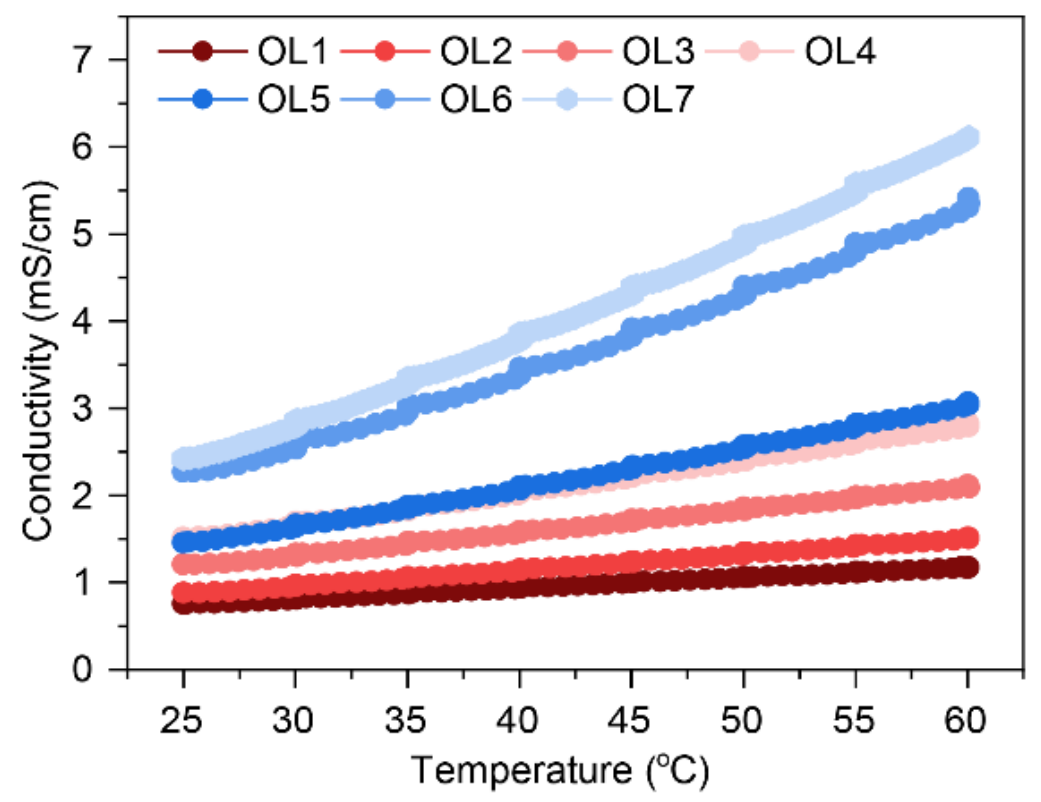

Figure S2. Ionic conductivities of $\mathrm{OL}$ electrolytes as a function of temperature from $25^{\circ} \mathrm{C}$ to $60^{\circ} \mathrm{C}$. 


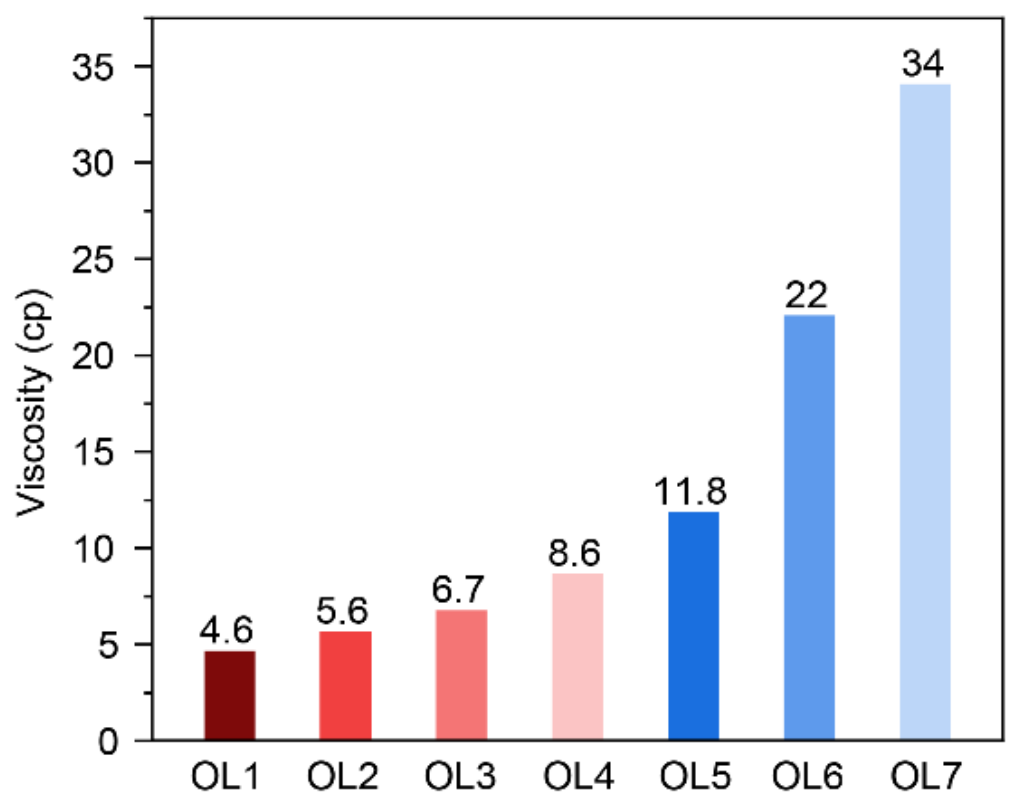

Figure S3. Viscosities of $\mathrm{OL}$ electrolytes at $25^{\circ} \mathrm{C}$.
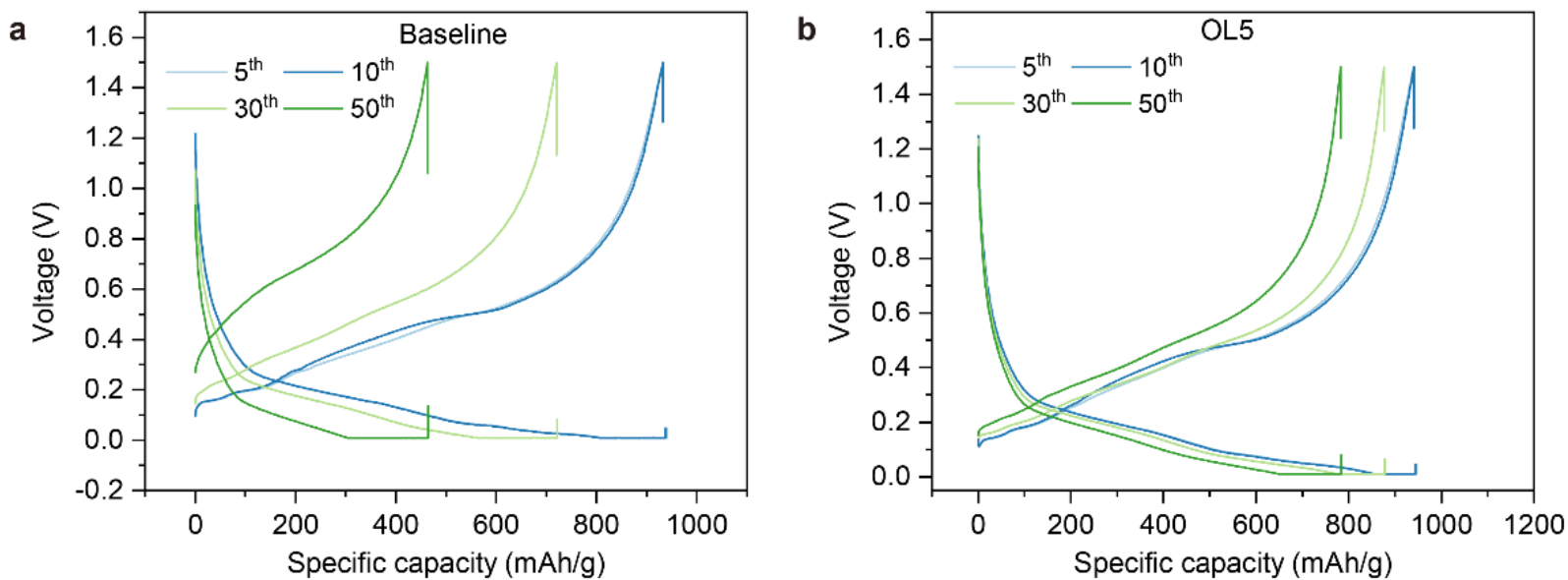

Figure S4. Voltage profiles of half-cells using the baseline (a) and OL5 (b) electrolytes at the 5th, 10th, 30th, and 50th cycles. 

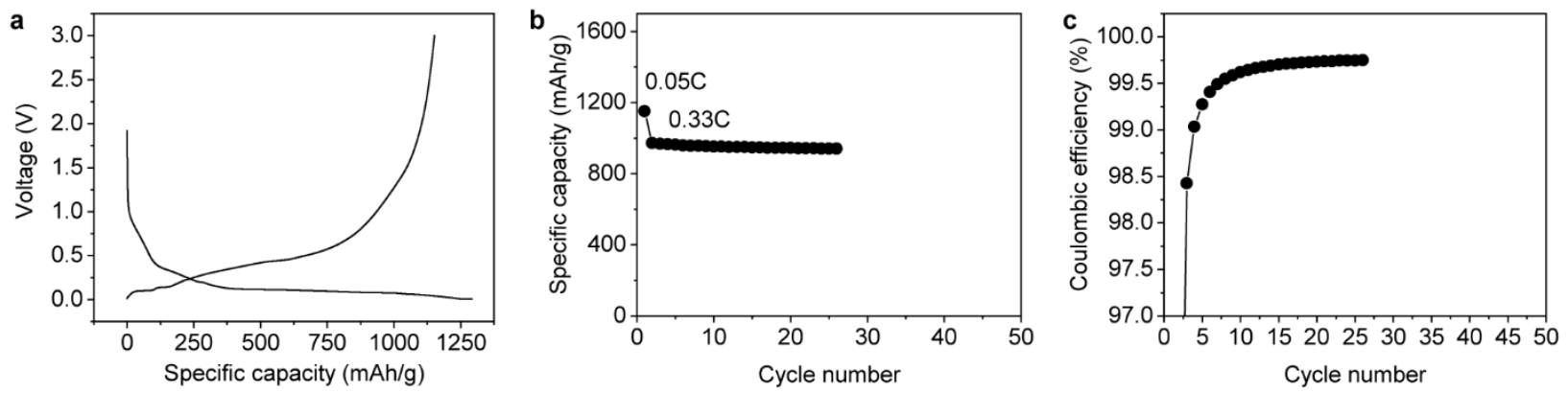

Figure S5. Electrochemical performance of Si/graphite electrode in the half-cell configuration. (a) Voltage profile of Si/graphite electrode using OL5 electrolyte without FEC and VC additives at the first cycle, b) Cycling performance and c) CE of Si/graphite electrode using OL5 electrolyte without FEC and VC additives.

The specific capacity and coulombic efficiency at the first cycle are in the same level with those with OL 5 electrolyte containing FEC and VC additives, i.e. specific capacity of 1152 $\mathrm{mAh} / \mathrm{g}$ with $89 \%$ coulombic efficiency. Moreover, the cycle life test under the same condition exhibits the capacity retention of $96.8 \%$ at $25^{\text {th }}$ cycle, which consolidates the superiority of OL electrolyte for stabilizing the Si/graphite anode. 


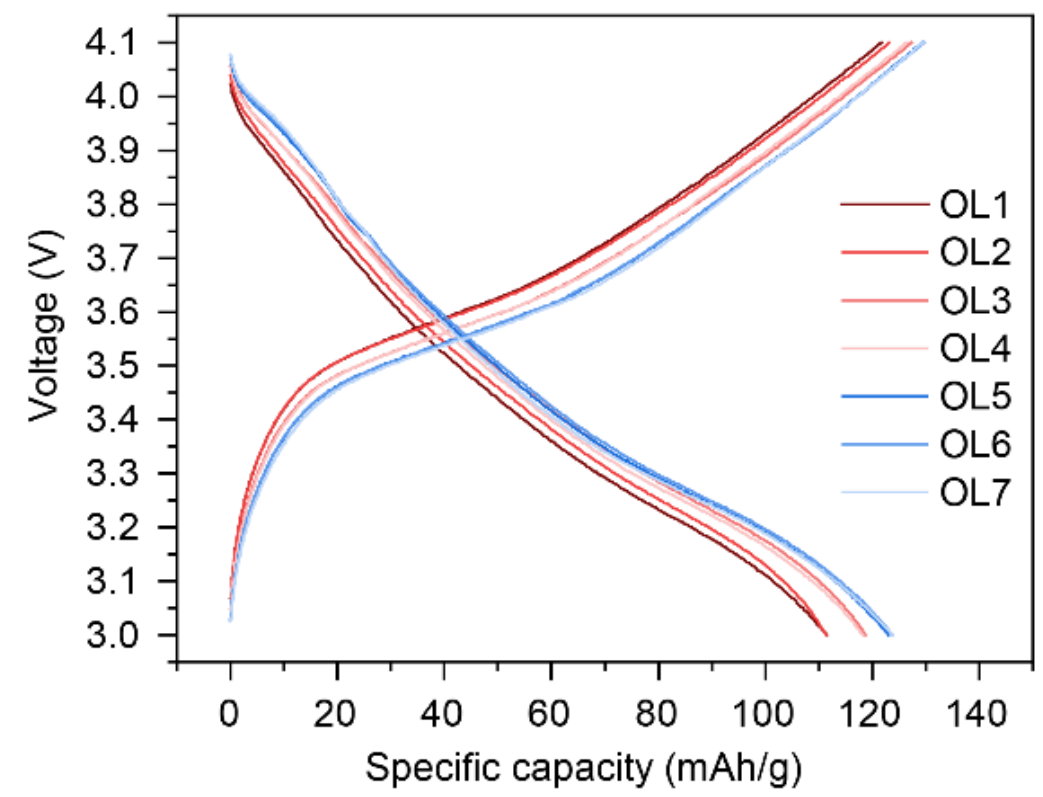

Figure S6. Voltage profiles of the full cells using different OL electrolytes at the first cycle.
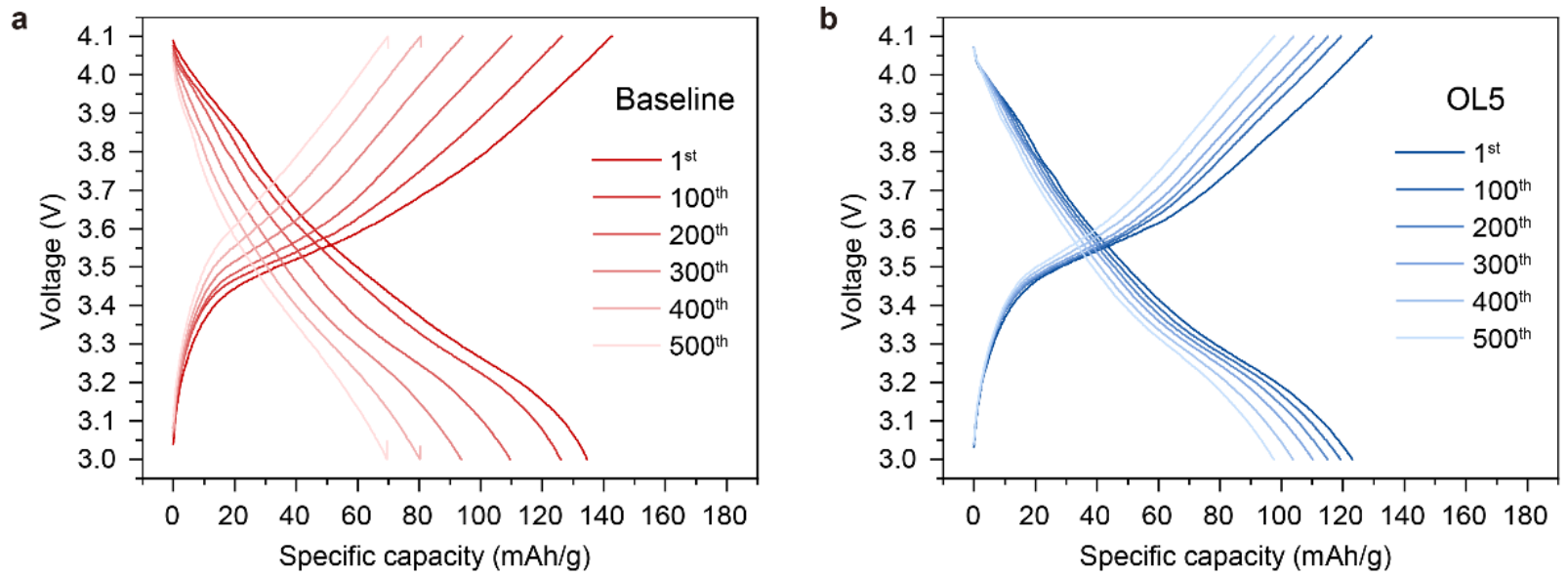

Figure S7. Voltage profiles of the full cell using the baseline (a) and OL5 (b) electrolytes at the 1st, 100th, 200th, 300th, 400th, and 500th cycles. 

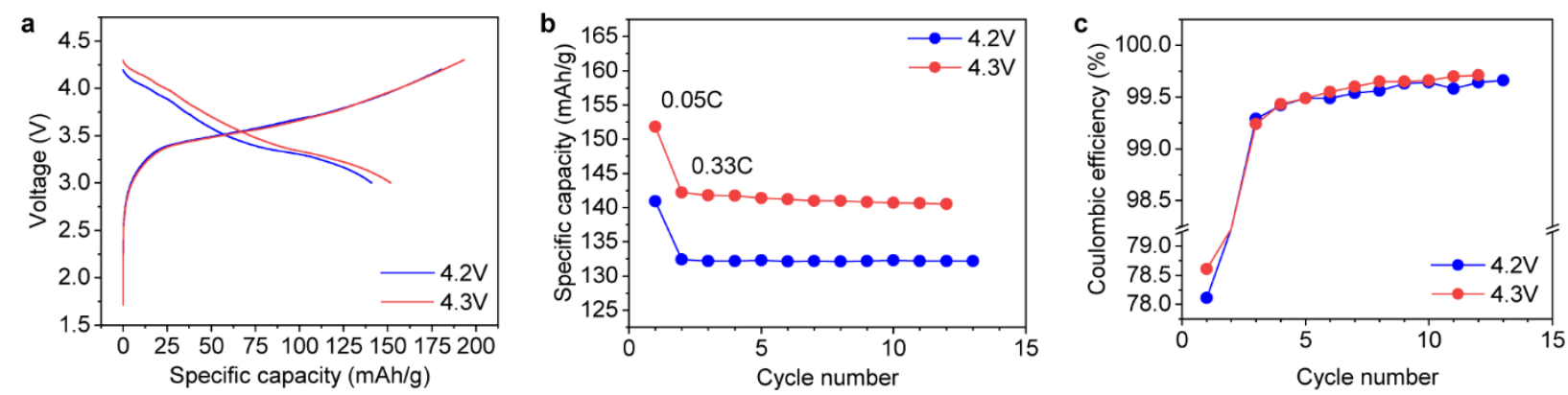

Figure S8. Electrochemical performance of Si/graphite||NMC532 full cells with the charge cutoff of 4.2 and $4.3 \mathrm{~V}$. (a) Voltage profile at the first cycle. (b) Cycling performance at the C-rate of $0.33 \mathrm{C}$ with the charge cutoff of 4.2 and $4.3 \mathrm{~V}$ (c) Coulombic efficiencies during cycling.
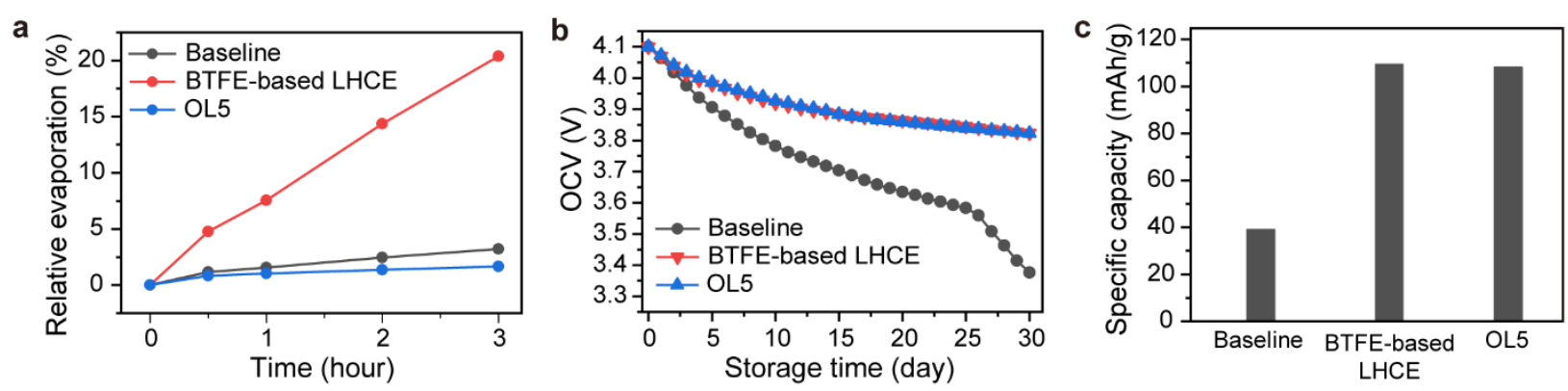

Figure S9. Storage and cycling performance of Si/graphite||NMC532 full cells at $45^{\circ} \mathrm{C}$.

(a) Relative evaporation of different electrolytes in a dry room. (b) Open-circuit voltage (OCV) drop of Si/graphite||NMC532 full cells during storage at $45^{\circ} \mathrm{C}$. (c) Discharge capacity after 30 days of storage at $45^{\circ} \mathrm{C}$.

Figure S9a shows the electrolyte evaporation rates of the baseline electrolyte and the two LHCE electrolytes measured in a dry room, where the room temperature and dew point were $20^{\circ} \mathrm{C}$ and $-42{ }^{\circ} \mathrm{C}$, respectively. Owing to the high boiling point of OTE diluent, OL5 evaporates substantially less than the BTFE-based LHCE and the baseline 
electrolyte, which contains EC (b. p. $243^{\circ} \mathrm{C}$ ) and EMC (b. p. $\left.107^{\circ} \mathrm{C}\right)$. This result implies that using OTE diluent can mitigate not only the electrolyte evaporation during battery manufacture but also the cell swelling from gas generation during cycling at high temperature.

Figure S9b shows the self-discharge behavior of the Si/graphite||NMC532 full cells with various electrolytes. The cells were charged to $4.1 \mathrm{~V}$ at $30{ }^{\circ} \mathrm{C}$ and then stored at $45^{\circ} \mathrm{C}$ over 30 days. After storage, the OCVs of the cells with LHCEs using BTFE or OTE as a diluent exhibit small OCV drops of $\sim 0.35 \mathrm{~V}$, much smaller than that with the baseline electrolyte $(0.75 \mathrm{~V})$. This is ascribed to the improved electrochemical stability of lithiated Si/graphite anodes against LHCEs at $45^{\circ} \mathrm{C}$, which subsequently is related to improvement of the SEI stability and electrolyte design. Figure S9c shows the capacity (at $0.05 \mathrm{C}$ ) and $\mathrm{CE}$ of the cells after the 30 days of storage. In accordance with the trend in OCV drop, the full cell using the baseline electrolyte exhibits only $39 \mathrm{mAh} / \mathrm{g}$ of specific discharge capacity as a result of self-discharge during the storage. But the BTFE-based LHCE and OL5 demonstrate specific discharge capacities of $109.4 \mathrm{mAh} / \mathrm{g}$ and $108.2 \mathrm{mAh} / \mathrm{g}$, respectively. These capacities are $27.6 \%, 81.2 \%$, and $81.8 \%$ of their charge capacities. These results indicate that the cells with OL5 exhibit much less capacity fade during storage than those with baseline electrolyte. Therefore, cells with OL5 will have much better calendar life than those with baseline electrolyte. 

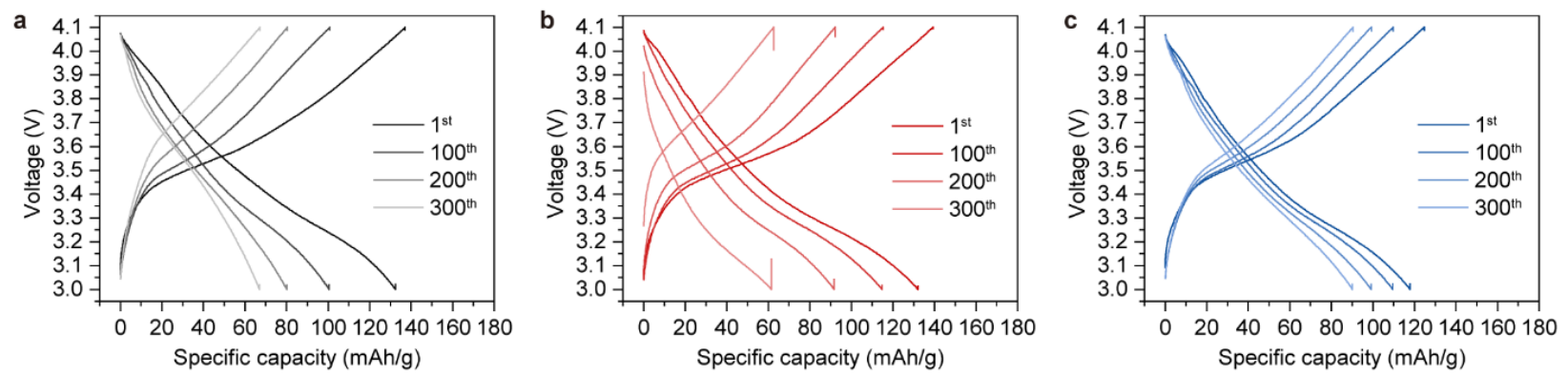

Figure S10. Voltage profiles of Si/graphite $\mid$ NMC532 full cells at the 1st, 100th, 200th, and 300th cycles using the baseline electrolyte (a), BTFE-based LHCE (b), and OL5 electrolyte (c).
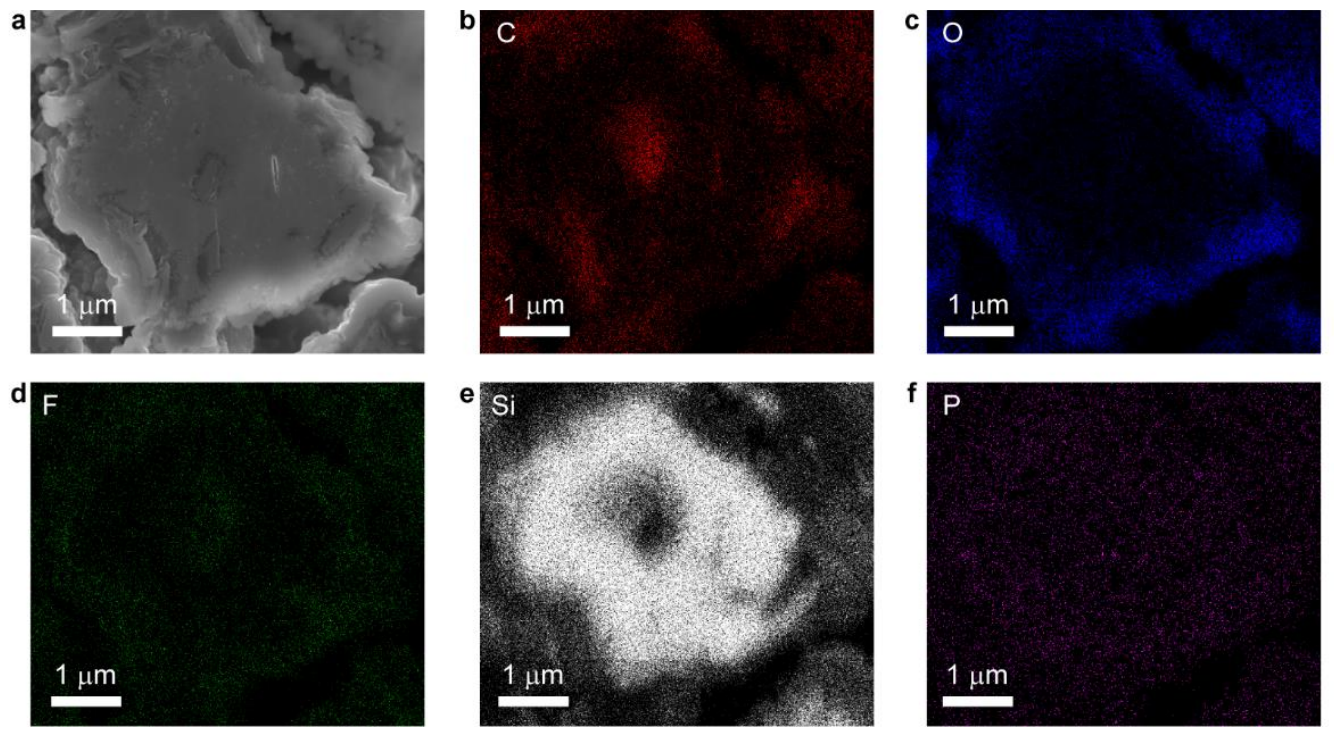

Figure S11. EDS mapping images of Si/graphite composite after 300 cycles with the baseline electrolyte in the cross-sectional view 

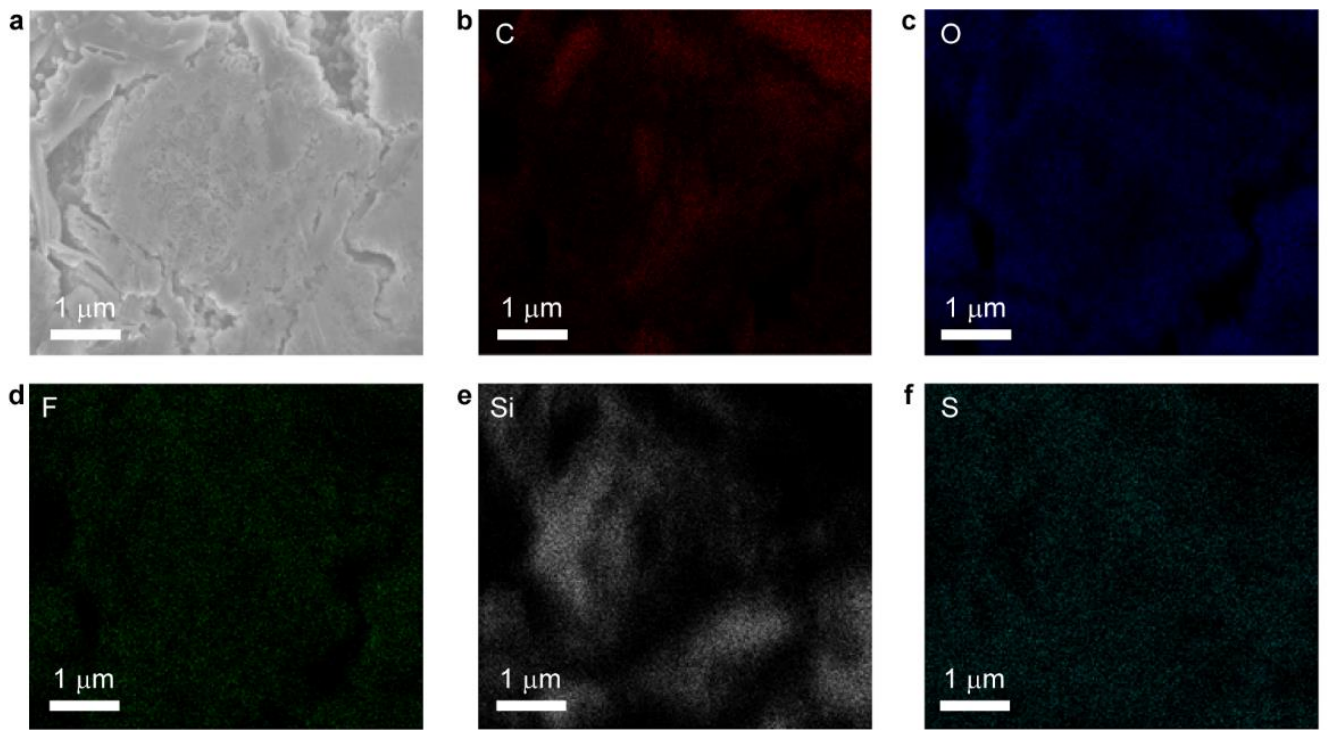

Figure S12. EDS mapping images of Si/graphite composite after 300 cycles with OL5 electrolyte in the cross-sectional view. 

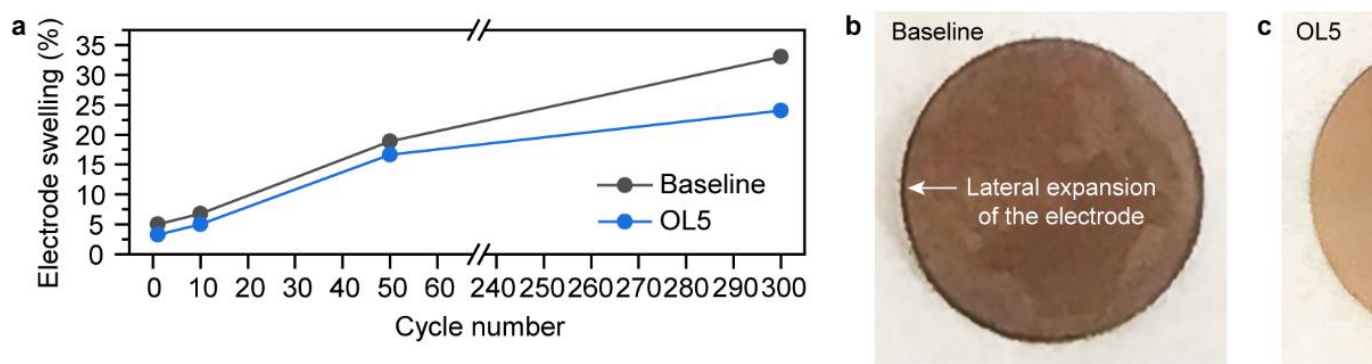

Figure S13. (a) Electrode swelling in the delithiated state upon cycling with the baseline and OL5 electrolytes. Digital photographs of the copper sides of cycled Si/graphite electrodes using the baseline (b) and OL5 (c) electrolytes. The electrode protrusion outside the current collector indicates the lateral swelling of the electrode under the high pressure of the spring in the coin cell.

To gauge the collective influence of SEI growth, the electrode swelling was measured after various cycles in full cells in delithiated states, as shown in Figure S11 (the measurement method is described in the experimental section). After short-term cycling (50 cycles), the anode cycled in OL5 electrolyte showed only slightly less swelling than that in the baseline electrolyte. This can be ascribed to the high initial porosity of the uncalendared electrodes ( 60\%). However, after 300 cycles, the electrode swelling with OL5 electrolyte $(24 \%)$ is much lower than that with the baseline electrolyte $(33 \%)$. Furthermore, lateral expansion of the electrode was observed after cycling in the baseline electrolyte (Figure S11 c). It can be ascribed to the combined effects of copious electrode swelling and high pressure from the spring of the coin-cell kit. This undesirable lateral expansion can cause delamination of the electrode in practical pouch or cylindrical cells, where a large jelly roll configuration is used. 

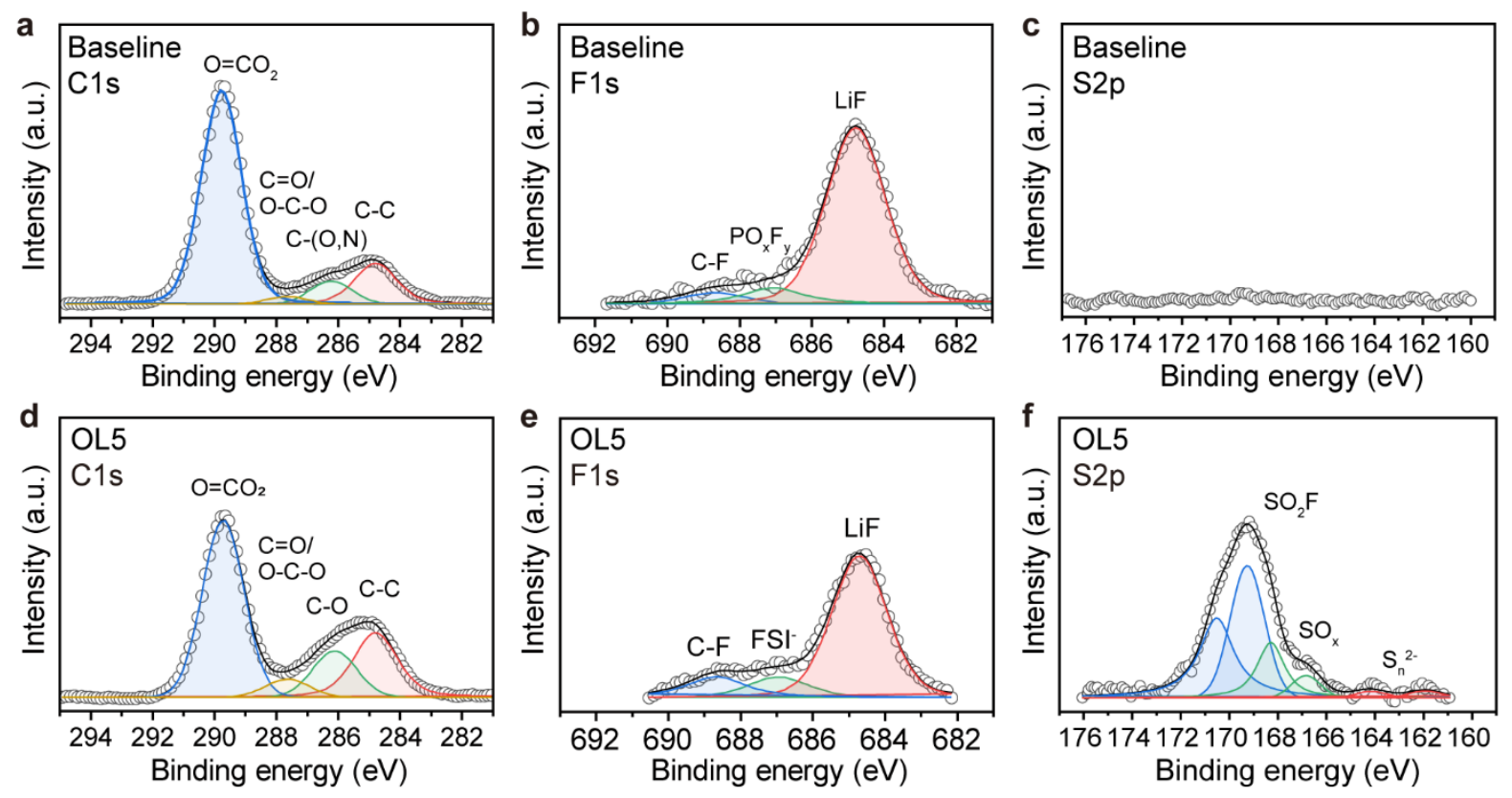

Figure S14. C 1s, F 1s, and S 2p spectra of Si/graphite anodes from XPS after 300 cycles with the baseline (a, b, c) and OL5(d, e, f) electrolytes.

Figure S14c-e and 14f-h show XPS C 1s, F 1s, S $2 p$ spectra on the SEI layers of Si/graphite composite cycled in the baseline and OL5 electrolytes, respectively. Comparing the spectra from the baseline and OL5 electrolytes, three remarkable features are evident. First, in the $\mathrm{C}$ 1s spectra, the relative intensity ratio of $\mathrm{O}=\mathrm{CO}_{2}$ to $\mathrm{C}-\mathrm{C}$ for $\mathrm{OL} 5$ electrolyte is higher than that for the baseline electrolyte. The $\mathrm{O}=\mathrm{CO}_{2}$ peaks are related to organic compounds of the SEI and the C-C peaks are related to the superficial carbon coating in the Si/graphite composite; this allows us to conclude that the accumulation of organic SEI compound during cycling on the surface of the Si/graphite composite is more restrained with OL5 electrolyte than with the baseline electrolyte. Second, for the F 1s spectrum, the relatively high intensities of $\mathrm{C}-\mathrm{F}$ and $\mathrm{FSI}^{-}$peaks from anodes cycled with 
OL5 electrolyte are regarded as SEI compounds from the decomposition of $\mathrm{FSI}^{-}$. Although the decomposition of $\mathrm{FSI}^{-}$also rapidly releases $\mathrm{F}^{-}$, forming $\mathrm{LiF}$ in the $\mathrm{SEI}$, the amount is less significant than that in the baseline electrolyte. High intensity of the LiF peak for the baseline electrolyte is related to the ready formation of $\mathrm{LiF}_{\text {from }} \mathrm{LiPF}_{6}$, as well as from leaching of the SEI layer by HF. ${ }^{1}$ Third, deconvolution of the $S 2 p$ spectrum from the Si anode cycled in OL5 provides direct evidence of the decomposition of $\mathrm{FSI}^{-}$, showing $\mathrm{SO}_{2} \mathrm{~F}, \mathrm{SO}_{x}$, and $\mathrm{Sn}^{2-}$, which are not observed in that of the baseline electrolyte. The sulfur in the SEI could improve the $\mathrm{Li}^{+}$conductivity of SEI layers, with the larger ionic radius and high atomic polarizability of the sulfide ions. ${ }^{2,3}$ In this regard, the SEI formed in OL5 electrolyte largely extended the cycle life of the Si/graphite anode.
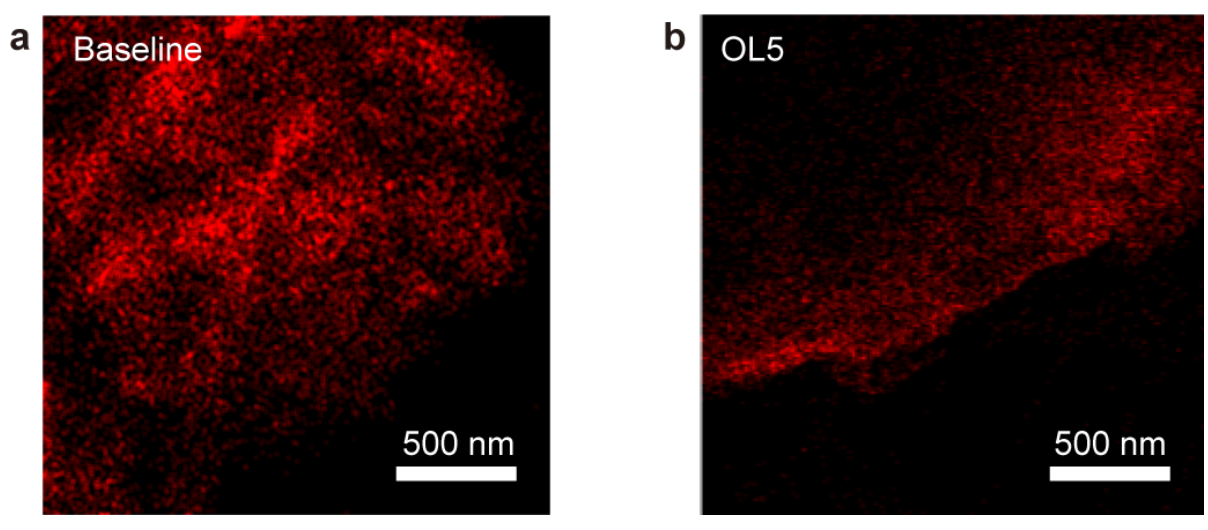

Figure S15. EDS mapping images of carbon for cycled Si/graphite anodes with the baseline (a) and OL5 (b) electrolytes.

\section{References}

(1) Shkrob, I. A.; Marin, T. W.; Zhu, Y.; Abraham, D. P., Why Bis(fluorosulfonyl)imide Is a "Magic Anion" for Electrochemistry. J. Phys. Chem. C 2014, 118, 19661-19671. 
(2) Piper, D. M.; Evans, T.; Leung, K.; Watkins, T.; Olson, J.; Kim, S. C.; Han, S. S.;

Bhat, V.; Oh, K. H.; Buttry, D. A.; Lee, S.-H., Stable Silicon-Ionic Liquid Interface for NextGeneration Lithium-Ion Batteries. Nat. Commun. 2015, 6, 6230.

(3) Chen, H.; Pei, A.; Lin, D.; Xie, J.; Yang, A.; Xu, J.; Lin, K.; Wang, J.; Wang, H.; Shi, F.; Boyle, D.; Cui, Y., Uniform High Ionic Conducting Lithium Sulfide Protection Layer for Stable Lithium Metal Anode. Adv. Energy Mater. 2019, 9, 1900858. 